Acta Cryst. (1997). D53, 269-273

\title{
Structure of the A-DNA Octamer d(GGCATGCC)
}

\author{
Christine M. NunN AND STEPHEN NEIDLE \\ The CRC Biomolecular Structure Unit, The Institute of Cancer Research, \\ Sutton, Surrey SM2 5NG, England. E-mail: c.nunn@icr.ac.uk \\ (Received 28 August 1996; accepted 27 November 1996)
}

\begin{abstract}
The crystal structure of the self-complementary DNA octamer d(GGCATGCC) has been determined to a resolution of $2.38 \AA$ and $R$ factor of $17.1 \%$. The structure is an A-DNA octamer duplex with Watson-Crick base pairing along its length. This sequence extends the investigation of octamer duplexes of the type $\mathrm{d}\left(\mathrm{GGX} \mathrm{X}_{4} \mathrm{CC}\right)$ which assume $P 6_{1}$ packing within the crystallographic unit cell. The A-DNA duplex is non-linear with a helix axis curvature of $7^{\circ}$ and bending towards the DNA minor groove. In view of the biological importance of protein recognition of some A-DNA sequences the minor-groove geometry and helix-axis curvature for this structure is compared with all others of the type $\mathrm{d}($ GGXATXCC).
\end{abstract}

\section{Introduction}

The structures of a number of native DNA octamer sequences have been determined by X-ray crystallography. With the exception of three sequences which contain alternating purine and pyrimidine bases and are consequently of Z-DNA type, all of these sequences crystallize as A-DNA duplexes. The vast majority of these A-DNA duplexes exist in two crystalline forms, in either hexagonal $P 6_{1}$ or tetragonal $P 4_{3} 2_{1} 2$ space groups. Whilst some sequences such as d(GGGCGCCC) exhibit both $P 6_{1}$ and $P 4_{3} 2_{1} 2$ crystal packing (Shakked, GuersteinGuzikevich, Eisenstein, Frolow \& Rabinovich, 1989), all of the known $P 6_{1}$ structures are of sequences of the type $\mathrm{d}\left(\mathrm{GGX} \mathrm{X}_{4} \mathrm{CC}\right)$ with the four central bases comprising $\mathrm{C}, \mathrm{G}, \mathrm{A}, \mathrm{T}, \mathrm{U}$ and $\mathrm{I}$.

Structural studies of protein-DNA complexes display a wide range of DNA conformations. For the structure of a TATA box-binding protein (TBP) complexed with a 14base-pair oligonucleotide (Kim, Geiger, Hahn \& Sigler, 1993; Kim, Nikolov \& Burley, 1993; Kim \& Burley, 1994) and the more recent structures of the transcription factor Ila with TBP and DNA (Tan, Hunziker, Sargent \& Richmond, 1996; Geiger, Hahn, Lee \& Sigler, 1996) the conformation of the DNA in these crystal structures has been found to be severely distorted from that of B-DNA type. In these structures the minor groove of the DNA has become wide and underwound to provide a large surface for interaction with the TBP assuming a conformation somewhat akin to features that one observes in A-DNA. Analysis of the conformation in the TATA box (Guzikevich-Guerstein \& Shakked, 1996) has shown that the conformation differs from canonical A-DNA by solely a rotation around each glycosidic bond along the DNA backbone. The structure of the TATA box containing sequence of the type $\mathrm{d}\left(\mathrm{GG} X_{4} \mathrm{CC}\right)$, where $X_{4}$ is TATA has been determined (Shakked et al., 1981) and is an A-DNA duplex, with $P 6_{1}$ packing within the unit cell. The close structural relationship shown for the TATA sequence in the native structure and upon protein binding highlights the importance of structural studies for sequences of this type.

In this paper the structure of the octamer sequence $\mathrm{d}(\mathrm{GGCATGCC})$ is presented. This sequence is of the type $\mathrm{d}\left(\mathrm{GG} X_{4} \mathrm{CC}\right)$ where $X_{4}$ is the alternating pyrimidine-purine sequence CATG. A recent structural study from this laboratory reported the structure of the decamer d(AGGCATGCCT) (Nunn \& Neidle, 1996) in which the central eight bases form an A-DNA octamer duplex.

\section{Materials and methods}

\subsection{Crystallization and data collection}

The DNA octamer d(GGCATGCC) was purchased from the Oswel DNA Service (University of Southampton, Southampton, UK). Crystals were grown by vapour diffusion at both 277 and $287 \mathrm{~K}$ from sitting drops containing $5 \mu \mathrm{l} 3 \mathrm{~m} M$ DNA in $30 \mathrm{~m} M$ sodium cacodylate $(\mathrm{pH} 7.0), 2 \mu \mathrm{l} 35 \%(v / v) \mathrm{NH}_{3}$ solution, $2 \mu \mathrm{l} 10 \mathrm{mM}$ hexamminecobalt(III) trichloride and $2 \mu \mathrm{l} 40 \%(v / v)$ 2-methyl-2,4-pentanediol, equilibrated against a $1 \mathrm{ml}$ reservoir of $40 \%(v / v)$ 2-methyl-2,4-pentanediol. Pale brown crystals grew with hexagonal cross-section within a few days, growing to an optimal size over a period of 7-10 d. A hexagonal prism grown at $287 \mathrm{~K}$ and measuring $0.4 \times 0.4 \times 0.4 \mathrm{~mm}$ was selected for data collection. Data collection was carried out using a Siemens-Xentronic multiwire area detector equipped with a rotating-anode $\mathrm{X}$-ray generator $(70 \mathrm{~mA}, 40 \mathrm{kV})$ and graphite monochromator. A crystal-to-detector distance of $10 \mathrm{~cm}$ and swing angle of $15^{\circ}$ was used. Data were collected with $\chi$ set at $45^{\circ}$, while the crystal was rotated through $100^{\circ}$ in $\omega .180$ second frames were recorded every $0.2^{\circ}$ steps.

Data processing was carried out using the program $X D S$ (Kabsch, 1993) and the CCP4 program suite (Collaborative Computational Project, Number 4, 1994). A 
Table 1. Helical parameters for d(GGCATGCC)

Calculated using program CURVES (Lavery \& Sklenar, 1988). Local parameters are shown in bold.

\begin{tabular}{|c|c|c|c|c|c|c|c|c|c|c|c|c|c|}
\hline Base pair & Tip $\left(^{\circ}\right)$ & Incline $\left(^{\circ}\right)$ & Buckle $\left({ }^{\circ}\right)$ & $\begin{array}{l}\text { Propeller } \\
\text { twist }\left({ }^{\circ}\right)\end{array}$ & Base step & \multicolumn{2}{|c|}{ Tilt $\left(^{\circ}\right)$} & \multicolumn{2}{|c|}{ Roll $\left(^{\circ}\right)$} & Twis & $\left(\left(^{\circ}\right)\right.$ & \multicolumn{2}{|c|}{$\begin{array}{l}\text { Base-pair } \\
\text { rise }(\dot{\mathrm{A}})\end{array}$} \\
\hline G1.C16 & 1 & 4 & -7 & 12 & G1.C16 & & & & & & & & \\
\hline G2.C15 & 1 & 6 & 6 & 10 & G2.C15 & 4 & 4 & 2 & 5 & 27 & 28 & 2.8 & 3.1 \\
\hline C3.G14 & 1 & 9 & 7 & -1 & C3.G14 & 1 & 1 & -4 & 0 & 32 & 32 & 3.4 & 3.5 \\
\hline A4.T13 & 1 & 11 & 2 & -4 & A4.T13 & -1 & -1 & 2 & 7 & 32 & 31 & 3.0 & 3.4 \\
\hline T5.A12 & 0 & 12 & 10 & 1 & T5.A12 & 2 & 2 & 0 & 7 & 32 & 31 & 2.9 & 3.3 \\
\hline G6.C11 & 1 & 13 & 0 & -4 & G6.C11 & 3 & 3 & 4 & 11 & 33 & 32 & 3.0 & 3.7 \\
\hline C7.G10 & 1 & 11 & -5 & 7 & C7.G10 & -1 & -2 & -2 & 5 & 31 & 30 & 3.2 & 3.6 \\
\hline C8.G9 & 2 & 12 & 5 & 5 & C8.G9 & 1 & o & 4 & 10 & 33 & 32 & 2.6 & 3.3 \\
\hline
\end{tabular}

Table 2. Backbone torsion angles $\left({ }^{\circ}\right)$ and furanose ring conformations for the octamer nucleotides

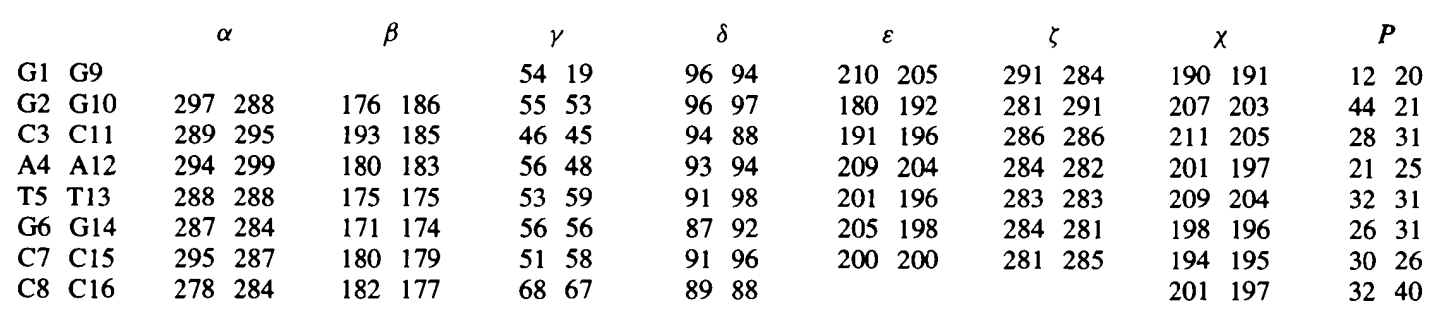

total of 5252 reflections were collected. Of a possible 2167 unique reflections, 2104 were observed to a resolution limit of $2.38 \AA$ representing a $97 \%$ complete data set. The final merged data set has cell parameters of $a=46.29, c=42.97 \AA$ and $R_{\text {merge }}(I)$ of $3.9 \%$. A second data set collected at $100 \mathrm{~K}$ using the same crystal showed a shrinkage in unit cell, however the resolution limit and crystal quality had deteriorated. This data was not used for the structure refinement.

\subsection{Structure refinement}

The structure crystallizes in the hexagonal space group $P 6_{1}$ with cell parameters close to those reported for a number of octameric sequences suggesting an isomorphous structure. These structures contain an octamer duplex within the asymmetric unit and are of A-DNA type. The coordinates for the structure of d(GGTATACC) (Shakked et al., 1981) were used as a starting model for the refinement with the central four bases replaced by those of the correct type.

Crystallographic refinement was carried out using the program $X-P L O R$ version 3.1 (Brünger, Kuriyan \& Karplus, 1987). Initial rigid-group refinement of the model as firstly one then 16 independent groups was carried out, followed by atomic positional refinement for data in the range $8-3 \AA$. The data range was extended to include data to the resolution limit of $2.38 \AA$ and in
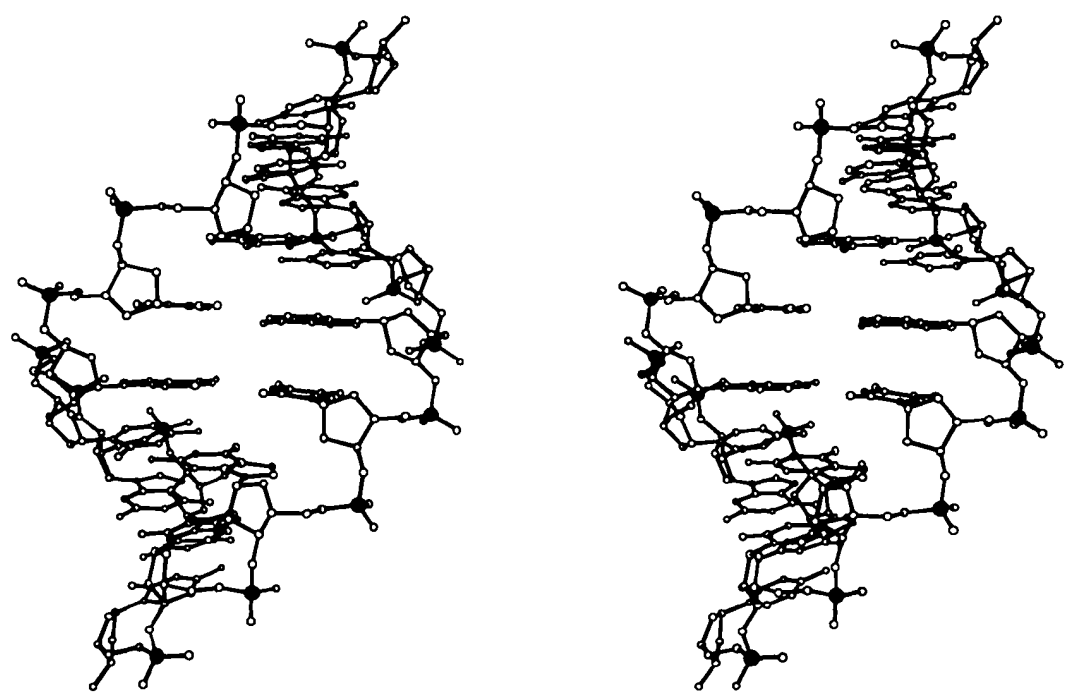

Fig. 1. Stereoview of the d(GGCATGCC) duplex. 
addition to positional refinement, atomic temperaturefactor refinement was carried out. After positional and temperature-factor refinement of all DNA atoms the $R$ factor was $21.7 \%$. Solvent molecules were located from difference electron-density maps and assigned as water molecules in the refinement. No hexamminecobalt(III) ions were located anywhere in the structure, and are presumably delocalized throughout the unit cell.

The refinement converged after the inclusion of 65 water molecules to give a final $R$ factor of $17.1 \%$ for data within the range $8-2.38 \AA$. Mean values for the thermal parameters $\left(\AA^{2}\right)$ are $27,26,26$, and 34 for the phosphate groups, sugars, bases and solvent molecules, respectively. The $X-P L O R$ refinement and $R$ factor calculations used 2045 data with $F>2 \sigma(F)$. The root-mean-square deviations from target values for bond lengths and angles are $0.009 \AA$ and $1.48^{\circ}$, respectively. The final atomic coordinates have been deposited in the Nucleic Acid Database.*

\section{Results and discussion}

The structure is an A-DNA octamer duplex (Fig. 1) comprising two self-complementary strands of the octamer within the crystallographic asymmetric unit with Watson-Crick base pairs along the entire length of the duplex. The bases are labelled $\mathrm{G} 1$ to $\mathrm{C} 8$ in the $5^{\prime}$ to $3^{\prime}$ direction for the first strand and G9 to C16 for the second. The number of base pairs per turn extrapolates to 11.3, which is typical for A-DNA. The helix axis is nonlinear with a total angle of curvature of $7.1^{\circ}$, bending in the central region of the helix towards the DNA minor groove.

The global and local (shown in bold) helical and basepair parameters for the octamer duplex are shown in Table 1. The terminal GpG step has a small helical twist with both of these G.C base pairs exhibiting large values of propeller twist. The propeller twist for the terminal two base pairs at each end of the duplex are larger than the central four base pairs. Backbone torsion angles for the octamer are similar along the length of the duplex (Table 2) and typical of values seen for A-DNA. Sugar puckers for the nucleotides are $\mathrm{C}^{\prime}$-endo for all but one guanosine (G2) and one cytosine $(\mathrm{C} 16)$ for which the pseudorotation angles are slightly larger and the conformation is $\mathrm{C4}^{\prime}$-exo. Intrastrand $\mathrm{P}-\mathrm{P}$ separations vary along the duplex, ranging from 5.7 to $6.3 \AA$ with the largest values occurring at the positions of $\mathrm{C} 4^{\prime}$-exo sugar pucker.

The stacking for each of the the dinucleotide steps for the octamer duplex is the same as that observed previously for A-DNA octamer duplexes of this type

\footnotetext{
* Atomic coordinates and structure factors have been deposited with the Nucleic Acid Database (Reference: ADH078, RADH078SF). Free copies may be obtained through The Managing Editor, International Union of Crystallography, 5 Abbey Square, Chester CH1 2HU, England (Reference: li0241).
}

(Lauble, Frank, Bloecker \& Heinemann, 1988). The terminal stacking interactions at both ends of the duplex show no stacking interactions between the cytosine bases and very little for the guanine bases. Stepping through the duplex the base-pair stacking shows alternating poor and good overlap, with good overlaps observed for the two GpC steps and the central ApT step - the (pyrimidine)p(purine) steps and poor overlaps for the other four steps. This feature is also seen in the structure of d(AGGCATGCCT) (Nunn \& Neidle, 1996). The DNA minor-groove width for d(GGCATGCC) is shown in Fig. 2 along with values for the corresponding sequence within the structure of d(AGGCATGCCT) (Nunn \& Neidle, 1996). The minor-groove width increases at the centre of the duplex though not to the degree shown for d(AGGCATGCCT).

The molecular packing of octamer duplexes within this $P 6_{1}$ cell has been observed previously for all other octameric sequences of the type $\mathrm{d}\left(\mathrm{GG} X_{4} \mathrm{CC}\right)$. Presumably this packing arrangement requires two contiguous guanine and cytosine bases at the $5^{\prime}$ and $3^{\prime}$ end of the sequence, respectively. The terminal base pair of one duplex stacks against the minor groove of a neighbouring duplex within the unit cell, related by either a $6_{1}$ or $2_{1}$ screw axis. There is only one intermolecular hydrogenbonding contact for d(GGCATGCC) from atom N2 of base $\mathrm{G}(2)$ to atom $\mathrm{O}^{\prime}$ ' of base $\mathrm{G}(9)$ of a symmetryrelated sugar phosphate backbone of $2.81 \AA$.

65 solvent sites were included in the refinement. All four of the terminal hydroxyl groups $105^{\prime}, 803^{\prime}, 905^{\prime}$ and $16 \mathrm{O}^{\prime}$ form hydrogen-bonding interactions with water molecules. Only limited hydration was observed in the DNA minor groove with water molecules directly bound to atom $\mathrm{N} 3$ of $\mathrm{A}(4)$ and $\mathrm{G}(10)$ and atom $\mathrm{O} 2$ of

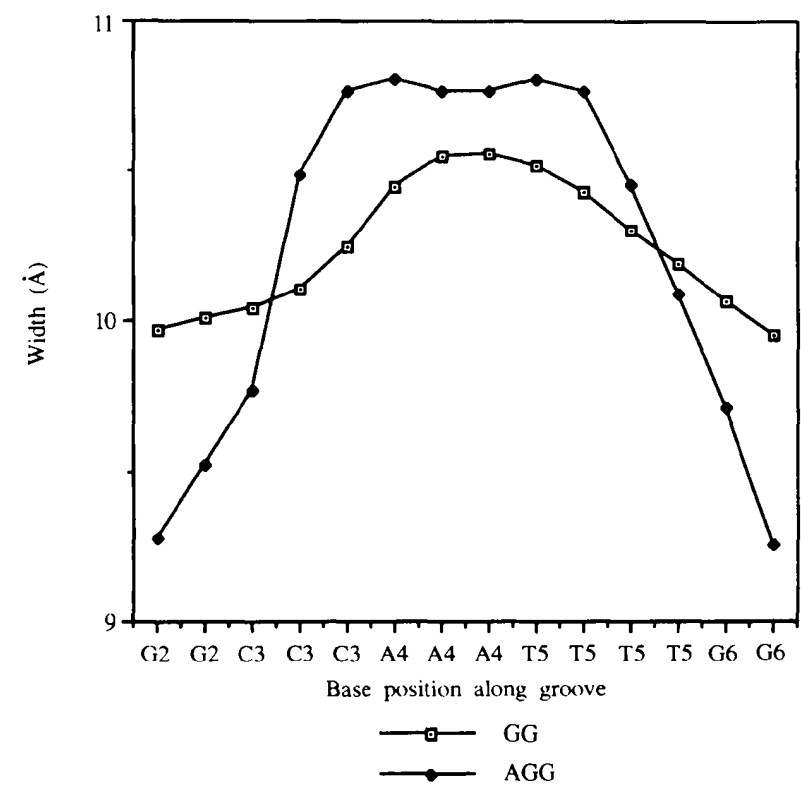

Fig. 2. Minor-groove width for d(GGCATGCC) together with the similar sequence in d(AGGCATGCCT) (Nunn \& Neidle, 1996). 
$\mathrm{C}(8), \mathrm{C}(11)$ and $\mathrm{C}(16)$. In the major groove the degree of hydration is greater with 11 solvent molecules directly hydrogen bonding to the DNA bases. The hydration of the phosphate groups is typical of A-DNA (Eisenstein \& Shakked, 1995) with 17 water molecules directly bound to phosphate $\mathrm{O}$ atoms. As a result of unit-cell packing the terminal base pairs $\mathrm{G}(1) \cdot \mathrm{C}(16)$ and $\mathrm{C}(8) \cdot \mathrm{G}(9)$ stack against symmetry-related duplexes. In this region of the

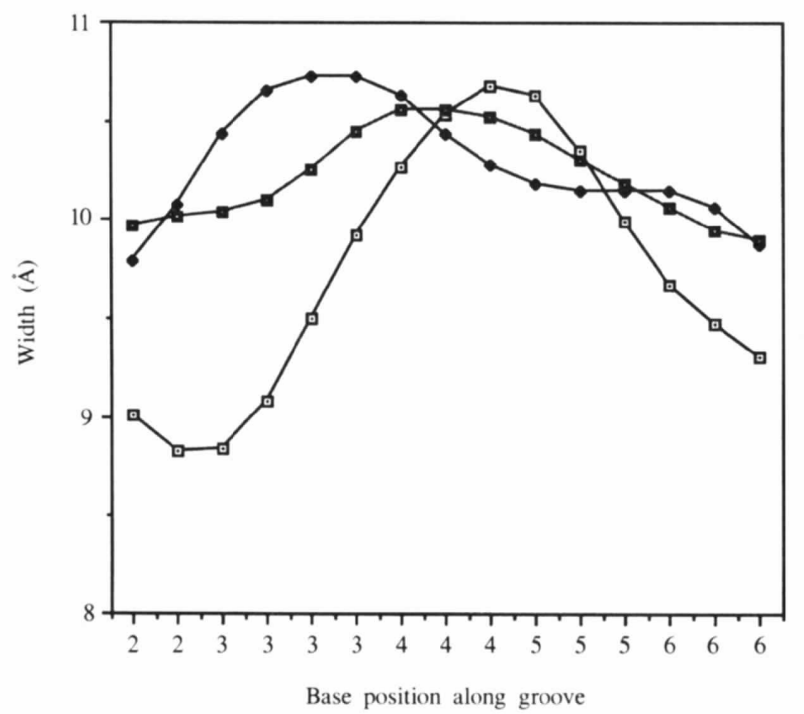

(a)

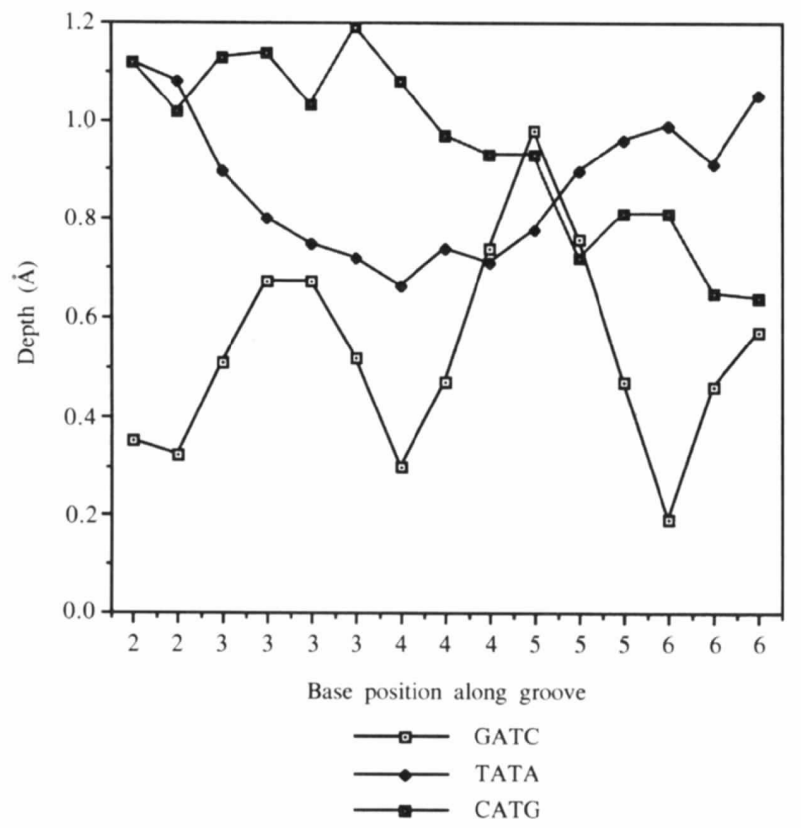

(b)

Fig. 3. Minor-groove width and depth for the structures of $\mathrm{d}$ (GGCATGCC), d(GGGATCCC) (Laube et al., 1988) and d(GGTATACC) (Shakked et al., 1983), shown as CATG, GATC and TATA, respectively. structure there are two solvent interactions between the adjacent minor grooves of the two symmetry-related helices. One water molecule binds to atom $\mathrm{O} 2$ of both $\mathrm{C}(11)$ and $\mathrm{C}(16)$ whilst there is a solvent-mediated interaction between atom $\mathrm{O} 2$ of $\mathrm{C}(8)$ and atom $\mathrm{N} 3$ of $\mathrm{A}(4)$.

The crystal structures of two other A-DNA octamer sequences with sequences close to d(GGCATGCC) have been reported. These sequences, d(GGGATCCC) (Lauble et al., 1988) and d(GGTATACC) (Shakked et al., 1983) both contain two base-pair changes from the present structure (shown as bold type). The sequence d(GGCATGCC) is close to being an alternating purine-pyrimidine sequence, with just one base-pair change at either end of the duplex compared to the Z-DNA octamer d(CGCATGCG) (Fujii et al., 1985). Another sequence which has six similar bases is d(GCATGCT) (Leonard et al., 1995). This sequence exhibits a quite different structure with a novel loop conformation which dimerizes to form a quadruplex structure.

The minor groove of A-DNA is recognised by proteins. It is of interest to examine the geometry of the DNA minor groove within the three similar $P 6_{1}$ A-DNA structures of the type $\mathrm{d}\left(\mathrm{GGX} X_{4} \mathrm{CC}\right)$ where $X_{4}=\mathrm{CATG}$, TATA and GATC. Because of the non-linear nature of the A-DNA helical axis in these structures the program CURVES (Lavery \& Sklenar, 1988) was used to investigate the minor-groove geometry (Stofer \& Lavery, 1994). The minor-groove width and depth for the three

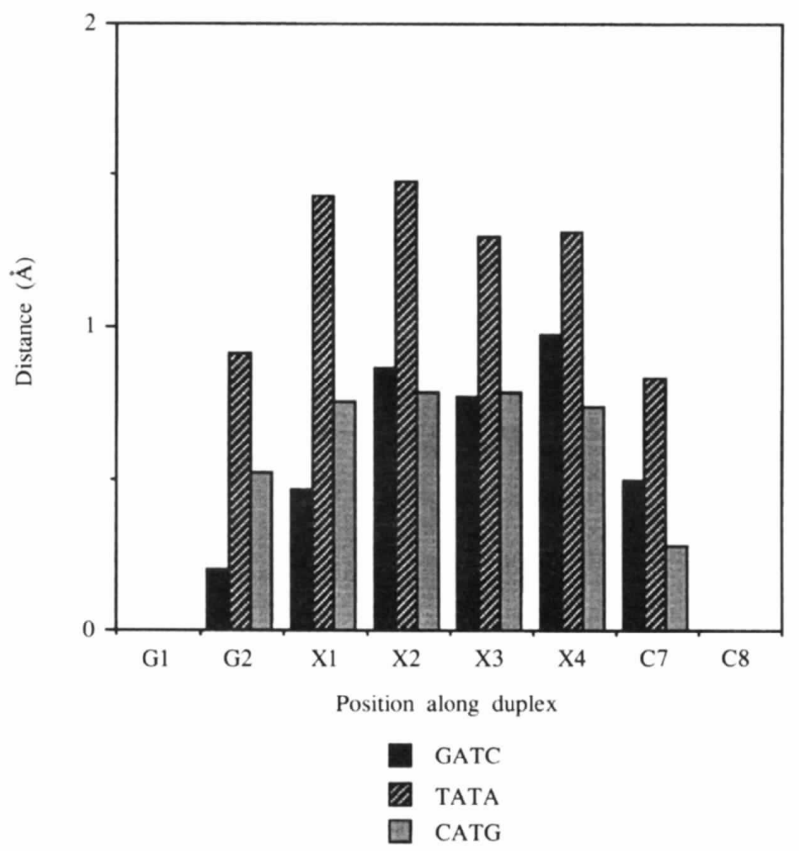

Fig. 4. Bar graph to show the base-pair offset from a straight line joining the terminal two base pairs for d(GGCATGCC), d(GGGATCCC) (Laube et al., 1988) and d(GGTATACC) (Shakked et al., 1983) octamer duplexes, shown as CATG, GATC and TATA, respectively. 
structures are shown in Fig. 3. The maximum groove width for all three structures is similar in magnitude, however the position of maximum groove width and variation across the groove differs for each sequence. The minor-groove depth for d(GGGATCCC) is largest at the position of maximum groove width. In the case of d(GGCATGCC) and d(GGTATACC) this does not hold and the minor-groove depth is much greater for these two structures.

In addition to the variation of minor-groove geometry observed for the $P 6_{1}$ octamer duplexes there is also marked variation in the curvature of the helix axis and the displacement of base pairs from the helix axis. Fig. 4 shows the offset of each base pair from a straight line joining the terminal two base pairs for the sequences d(GGCATGCC), d(GGGATCCC) and d(GGTATACC). The TATA sequence, which is known to undergo minorgroove binding with protein molecules and is present in a wide range of promoter sequences shows a large axis curvature with bending of $21^{\circ}$ towards the DNA minor groove. The sequence d(GGGATCCC) containing a shorter AT region at the centre of the duplex exhibits much less helix curvature with bending of $11^{\circ}$, close to the value seen here for d(GGCATGCC) of $7^{\circ}$.

These results show that the central four base pairs within these octamer duplexes are conformationally variable within A-DNA structure and do not impose a rigid helical geometry. In relation to protein binding this DNA flexibility enables DNA and protein molecules to potentially interact with maximum interaction.

For the octamer duplex observed in the structure of d(AGGCATGCCT) (Nunn \& Neidle, 1996) the helixaxis curvature is $17^{\circ}$ towards the minor groove. The increased curvature for this structure is probably a result of crystal packing effects and base-pairing interactions of the terminal $\mathrm{A}$ and $\mathrm{T}$ bases. Within this structure cobalt hexammine ions bind to the DNA major groove via hydrogen-bonding interactions with $\mathrm{GG}$ bases at each end of the duplex in addition to a second binding site with backbone phosphate groups. Bending of DNA as a result of charge neutralization is known to occur
(Crothers, 1994) and could also explain the increase in curvature that we observe in this structure.

\section{References}

Brünger, A. T., Kuriyan, J. \& Karplus, M. (1987). Science, 235, 458-460.

Collaborative Computational Project, Number 4 (1994). Acta Cryst. D50, 760-763.

Crothers, D. M. (1994). Science, 266, 1819-1820.

Eisenstein, E. \& Shakked, Z. (1995). J. Mol. Biol. 248, 662-678.

Fujii, S., Wang, A. H.-J., Quigley, G. J., Westerink, H., van Der Marel, G. A., van Boom, J. H. \& Rich, A. (1985). Biopolymers, 24, 243-250.

Geiger, J. H., Hahn, S., Lee, S. \& Sigler, P. B. (1996). Science, 272, 830-836.

Guzikevich-Guerstein, G. \& Shakked, Z. (1996). Nature Struct. Biol. 3, 32-37.

Kabsch, W. (1993). J. Appl. Cryst. 26, 795-800.

Kim, J. L. \& Burley, S. K. (1994). Nature Struct. Biol. 1, 638-653.

Kim, J. L., Nikolov, D. B. \& Burley, S. K. (1993). Nature (London), 365, 520-527.

Kim, Y., Geiger, J. H., Hahn, S. \& Sigler, P. B. (1993). Nature (London), 365, 512-520.

Lauble, H., Frank, R., Bloecker, H. \& Heinemann, U. (1988). Nucleic Acids Res. 16, 7799-7816.

Lavery, R. \& Sklenar, H. (1988). J. Biomol. Struct. Dynam. 6, 63-91.

Leonard, G. A., Zhang, S., Peterson, M. R., Harrop. S. J., Helliwell, J. R., Cruse, W. B. T., d'Estaintot, B. L., Kennard, O., Brown, T. \& Hunter, W. N. (1995). Structure, 3, 335-340.

Nunn, C. M. \& Neidle, S. (1996). J. Mol. Biol. 256, 340-351.

Shakked, Z., Guerstein-Guzikevich, G., Eisenstein, M., Frolow, F. \& Rabinovich, D. (1989). Nature (London), 342, 456-460. Shakked, Z., Rabinovich, D., Cruse, W. B. T., Egert, E., Kennard, O., Sala, G., Salisbury, S. A. \& Viswamitra, M. A. (1981). Proc. R. Soc. London Ser. B, 213, 479-487.

Shakked, Z., Rabinovich, D., Kennard, O., Cruse, W. B. T., Salisbury, S. A. \& Viswamitra, M. A. (1983). J. Mol. Biol. 166, 183-201.

Stofer, E. \& Lavery, R. (1994). Biopolymers, 34, 337-346.

Tan, S., Hunziker, Y., Sargent, D. F. \& Richmond, T. J. (1996). Nature (London), 381, 127-134. 